\title{
Switching classical trajectory Monte Carlo method to describe two-active-electron collisions
}

\author{
A. Jorge, ${ }^{1}$ Clara Illescas, ${ }^{1}$ L. Méndez, ${ }^{1}$ and B. Pons ${ }^{2}$ \\ ${ }^{1}$ Laboratorio Asociado al CIEMAT de Física Atómica y Molecular en Plasmas de Fusión, Departamento de Química, Módulo 13, \\ Universidad Autónoma de Madrid, Cantoblanco E-28049 Madrid, Spain \\ ${ }^{2}$ Université de Bordeaux, CNRS, CEA, CELIA, UMR5107, F-33405 Talence, France
}

(Received 31 May 2016; published 17 August 2016)

\begin{abstract}
We propose a classical trajectory Monte Carlo method to describe two-center collisions with two active electrons. The approach is based on switching between standard four-body and three-body descriptions and it is therefore easy to implement. We demonstrate the reliability of the approach for fundamental $\mathrm{H}+\mathrm{H}$ and $\mathrm{H}^{+}+\mathrm{H}^{-}$ collisions that neither four-body nor three-body classical methods describe satisfactorily.
\end{abstract}

DOI: 10.1103/PhysRevA.94.022710

Abrines and Percival introduced 50 years ago a statistical classical approach to atomic collisions [1], which since then has become one of the most useful methods to calculate cross sections and to picture the mechanisms underlying inelastic processes (see, e.g., [2-6] and references therein). This approach, which employs classical mechanics to describe nonadiabatic dynamics in terms of noninteracting electron trajectories, is referred to as the classical trajectory Monte Carlo (CTMC) method. Beyond atomic collisions, CTMC is also widely used in the framework of laser-matter interactions [7-12]. However, the applicability of CTMC is usually restricted to one-active-electron dynamics, since many-electron systems are classically unstable and subject to artificial autoionization (see [13] and references therein). Therefore, many-electron systems are generally described within the independent electron model [14,15], employing effective charges [16,17] or static model potentials [18-20] to represent the interaction of one active electron with the ionic core, which is assumed to remain frozen during the interaction. This model yields satisfactory agreement with experimental data for one-electron processes [3,16], but multielectron processes are generally overestimated [18,20,21]. The overestimation has been interpreted as a result of the frozen ionic core approximation, and a dynamical screening approach, in which the interaction between active electrons is represented in terms of a time-dependent model potential, was then proposed [22]. It led to an improvement of computed double-ionization cross sections in $\mathrm{p}+\mathrm{He}$ collisions. A similar improvement was achieved in [13], where the electron-electron interaction was softened by describing the active electrons in terms of Gaussian density distributions. Alternatively to these screening approaches, artificial autoionization can be prevented by adding to the electronic Hamiltonian a repulsive potential which prevents the electrons approaching too close to each other [10,23-25].

In this work, we propose a CTMC method for two-activeelectron systems, called switching CTMC. It combines the usual static screening approach, which is employed when the two electrons are bound to the same nuclear center, with an exact description of the electron-electron interaction as soon as electron detachment occurs. Our approach has the main advantages of CTMC, i.e., it is easy to implement and provides a picture of the dynamics, but, in contrast to all previous approaches, our method is free from any additional parameter that constrains the electronic repulsion, apart from the wellestablished form of model potentials for static screening. In the present work, we illustrate the reliability of our approach for the two fundamental collision systems, $\mathrm{H}(1 s)+\mathrm{H}(1 s)$ and $\mathrm{H}^{+}+\mathrm{H}^{-}$, as prototypes of collisions where active electrons are initially located either on different centers or on the same nucleus. Besides its application to these fundamental two-center systems, the method can be easily extended to any other two-electron process taking place, e.g., in ion-cluster collision or laser-atom or -molecule interaction.

$\mathrm{H}(1 s)+\mathrm{H}(1 s)$ collisions have been investigated experimentally [26-28]. These experiments reported total cross sections for electron capture by the projectile, also called negativeprojectile ion formation:

$$
\mathrm{H}\left(e_{2}\right)+\mathrm{H}\left(e_{1}\right) \rightarrow \mathrm{H}^{-}\left(e_{1}, e_{2}\right)+\mathrm{H}^{+} .
$$

Particular attention has been paid to this electron capture reaction from the theoretical side; it has been studied by means of semiclassical close coupling [29-31], perturbative first Born [32-35], and four-body CTMC (4b-CTMC) [36-38] approaches. The 4b-CTMC calculations of Becker and McKellar [37] drastically underestimated the cross section for reaction (1). The authors explained this result as a consequence of the very small electronic phase space for classical $\mathrm{H}^{-}$, as previously pointed out by Olson (private communication in [37]), who showed that the trajectories leading to $\mathrm{H}^{-}$formation autodetach as the integration time increases. However, the 4b-CTMC calculations of Olson [36] overestimate the experimental cross section for reaction (1). A more recent 4b-CTMC calculation, by Dimitriou et al. [38], has yielded a clear underestimation of this cross section, as expected from artificial autoionization that follows the formation of the classical $\mathrm{H}^{-}$, and in agreement with the findings of Becker and McKellar.

$\mathrm{H}^{+}+\mathrm{H}^{-}$collisions have been studied extensively, from both the experimental [39-44] and theoretical [30,31,45-52] sides. In this respect, the main challenges of a classical description are to describe a stable two-electron target anion and to correctly represent the mutual neutralization process,

$$
\mathrm{H}^{+}+\mathrm{H}^{-}\left(e_{1}, e_{2}\right) \rightarrow \mathrm{H}(n l m)+\mathrm{H}\left(n^{\prime} l^{\prime} m^{\prime}\right),
$$

in which electron correlation effects may play an important role. Theoretical treatments usually assume that neutralization involves only the loosely bound electron of $\mathrm{H}^{-}$and therefore 
employs a static model potential to represent the interaction of this active electron with the frozen ionic core. This reduces the original four-body problem to a simpler three-body system [47,48,51]. However, some works [47,51] suggest that this description is not valid at high collision energies where an important contribution to neutralization comes from the core electron.

We consider first the 4b-CTMC approach to explain its limitations to reliably describe the $\mathrm{H}\left(e_{2}\right)+\mathrm{H}\left(e_{1}\right)$ reaction. The Hamiltonian of the $4 \mathrm{~b}-\mathrm{CTMC}$ treatment includes the unscreened Coulomb forces between the four bodies, the two electrons, and the two point charges for the nuclei. Since the two electrons are initially bound to different nuclei, the initial electron distribution consists of the product of two microcanonical distributions, $\delta\left[H\left(e_{1}\right)-E_{0}\right] \delta\left[H\left(e_{2}\right)-E_{0}\right]$, where $H\left(e_{i}\right)=p_{i}^{2} / 2-1 / r_{i}$ is the Hamiltonian of electron $e_{i}$, with $\mathbf{r}_{i}$ and $\mathbf{p}_{i}$ its position and momentum with respect to the proton to which it is bound, and $E_{0}=-0.5$ a.u. In practice, the microcanonical ensembles are built according to the original recipe of Abrines and Percival [1], and they both contain the same number of independent trajectories $\mathcal{N}=3100$. For a nuclear trajectory initially characterized by the impact velocity $\mathbf{v}$ and the impact parameter $\mathbf{b}$, the twoelectron dynamics are obtained by integrating the Hamilton equations for the $\mathcal{N}^{2}$ pairs of electrons up to the final time $t_{\text {fin }}=2000 / v$ a.u. For each electron pair, we calculate the one-electron energies with respect to the projectile $\left(E_{1,2}^{(\mathrm{P})}\right)$ and the target $\left(E_{1,2}^{(\mathrm{T})}\right)$ at $t=t_{\text {fin }}$. The elastic and excitation processes correspond to $E_{i}^{(\mathrm{P})}<0$ and $E_{j}^{(\mathrm{T})}<0(i \neq j)$. For the singleionization process one has $E_{i}^{(\mathrm{P})}>0$ and $E_{i}^{(\mathrm{T})}>0$ with either $E_{j}^{(\mathrm{P})}<0$ or $E_{j}^{(\mathrm{T})}<0$, while for the double-ionization process, all the monoelectronic energies $E_{1,2}^{(\mathrm{P}),(\mathrm{T})}$ are positive. For the electron capture process, the electron energies fulfill either $E_{\text {tot }}^{(\mathrm{P})}=E_{1}^{(\mathrm{P})}+E_{2}^{(\mathrm{P})}+1 / r_{12}<0$, with $E_{1}^{(\mathrm{P})}<0$ and $E_{2}^{(\mathrm{P})}<0$ (projectile $\mathrm{H}^{-}$formation), or $E_{\mathrm{tot}}^{(\mathrm{T})}=E_{1}^{(\mathrm{T})}+E_{2}^{(\mathrm{T})}+1 / r_{12}<0$, with $E_{1}^{(\mathrm{T})}<0$ and $E_{2}^{(\mathrm{T})}<0$ (target $\mathrm{H}^{-}$formation).

The main failure of the standard 4b-CTMC approach lies in the description of the electron capture process. As already mentioned, whenever a two-electron $\mathrm{H}^{-}\left(e_{1}, e_{2}\right)$ anion is formed along the collision, autoionization takes place as soon as the two orbiting electrons come too close to each other. The delay between $\mathrm{H}^{-}$formation and artificial electron detachment depends on the orbiting conditions. However, autoionization inevitably occurs so that the probability for $\mathrm{H}^{-}$ formation continuously decreases as time increases, as shown in Fig. 1 for a prototypical nuclear trajectory with $E=30 \mathrm{keV}$ and $b=1$ a.u. The probability for $\mathrm{H}^{-}$formation vanishes down to 0 , leading simultaneously to an overestimation of genuine single-electron ionization probabilities.

Our approach to circumvent this drawback consists in switching between standard 4b- and 3b-CTMC methods. We employ $4 \mathrm{~b}-\mathrm{CTMC}$ as long as the two active electrons are not bound to the same nuclear center, and we switch to 3b-CTMC, with static screening, as soon as the two electrons bind to the same nucleus with either $\left[E_{1}^{(\mathrm{T})}(t)<0, E_{2}^{(\mathrm{T})}(t)<0, E_{\text {tot }}^{(\mathrm{T})}(t)<\right.$ $0]$ or $\left[E_{1}^{(\mathrm{P})}(t)<0, E_{2}^{(\mathrm{P})}(t)<0, E_{\text {tot }}^{(\mathrm{P})}(t)<0\right]$. Nevertheless, we still consider explicitly the dynamics of the two electrons after switching by considering simultaneously, but independently,

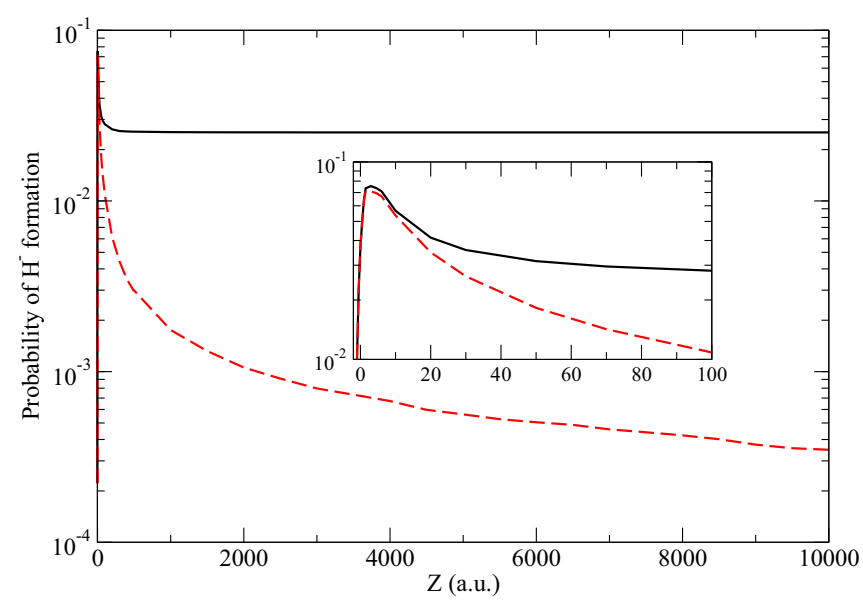

FIG. 1. Evolution of electron capture transition probability as a function of $Z=v t$ in $\mathrm{H}(1 s)+\mathrm{H}(1 s)$ collisions with $E=30 \mathrm{keV}$ and $b=1$ a.u. Results from switching (-) and $4 \mathrm{~b}$ (- - -) CTMC calculations. In the inset, we zoom in on the $-5 \leqslant Z \leqslant 100$ a.u. interval.

the two three-body systems $\left\{\left(\mathrm{H}^{+}+e_{2}\right)+e_{1}\right\}+\mathrm{H}^{+}$and $\left\{\left(\mathrm{H}^{+}+e_{1}\right)+e_{2}\right\}+\mathrm{H}^{+}$. Dynamics of electron $e_{1}$ are represented by the former $3 \mathrm{~b}$ system, where the electron $e_{2}$ is not active, while the dynamics of electron $e_{2}$ are followed in terms of the later $3 \mathrm{~b}$ system with inactive $e_{1}$. In practice, the interaction between the subsystem $\left(\mathrm{H}^{+}+e_{i}\right)$ and the active electron $e_{j}$, with $\{i, j\}=\{1,2\}$, is described by the static model potential $V_{\text {mod }}\left(r_{j}\right)=-\left(1+\alpha r_{j}\right) \exp \left(-2 \alpha r_{j}\right) / r_{j} \quad[49,53]$, where $\alpha=$ 0.65 (a.u. $^{-1}$ is chosen to yield the first ionization potential of $\mathrm{H}^{-}, 0.027$ a.u. When the active electron of one of the $3 \mathrm{~b}$-systems is freed through ionization or electron capture, we switch back to the propagation of the $4 \mathrm{~b}$ system with explicit consideration of the electron-electron repulsion term $1 / r_{12}$. The changes in the potentials felt by each electron at the moment of switching from the $4 b$ - to the $2 \times 3 b-$ CTMC calculations (or vice versa) are smooth and do not cause numerical instabilities. This is illustrated in the upper panel of Fig. 2, where we plot the temporal evolution of the electron distances $r_{1}$ and $r_{2}$ measured with respect to the target nucleus for a representative trajectory with $E=30 \mathrm{keV}$ and $b=1$ a.u. that leads to negative target ion formation. One can note that $r_{1}$ and $r_{2}$ behave smoothly around $t=0$ a.u., where we switch from $4 \mathrm{~b}$ - to $2 \times 3 \mathrm{~b}-\mathrm{CTMC}$ propagations because of $\mathrm{H}^{-}$formation. For $t>0$, the static screening description of the electron-electron repulsion ensures that the target anion remains stable, and therefore we obtain (Fig. 1) a capture probability that stabilizes for $t \lesssim 100$ a.u., contrary to what happened with the 4b-CTMC approach. This stability for the $\mathrm{H}^{-}$is clearly observed in the lower panels of Fig. 2, where the classical orbits of the two electrons are shown, before and after the switching. These panels illustrate the different nature of the orbits associated to Coulomb and model potentials for $\sim 50 / v$ a.u. of integration time, around the nucleus they are bound to. Similar plots are found for all the electron pairs that lead to negative target (and projectile) ion formation.

We present in Fig. 3 the total cross section for negativeprojectile ion formation [Eq. (1)], calculated by means of 

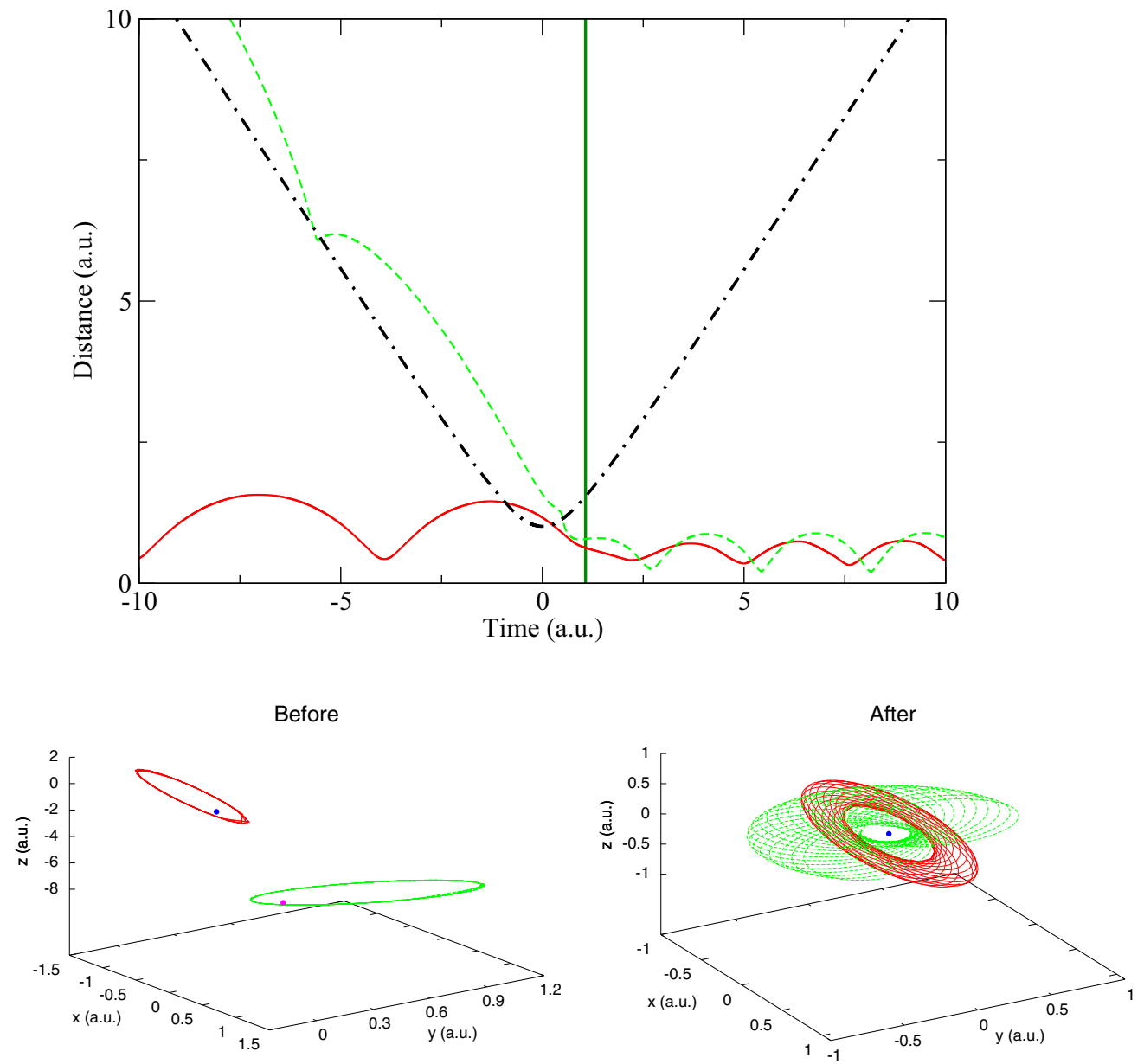

FIG. 2. Upper panel: Illustration of the switching between $4 \mathrm{~b}-$ and $2 \times 3 \mathrm{~b}$ CTMC calculations in $\mathrm{H}(1 s)+\mathrm{H}(1 s)$ collisions. The figure shows the time evolution of electron distances, $r_{1}$ (full line) and $r_{2}$ (dashed line), to the target nucleus, and the distance from the projectile to the target nucleus (dashed-dotted line), for a trajectory initially defined by $E=30 \mathrm{keV}$ and $b=1$ a.u. that leads to $\mathrm{H}^{-}$formation. The vertical line indicates the time when the switching takes place. Lower panels: Classical electron orbits before $(-50<t<-5$ a.u. $)$ and after $(5<t<50$ a.u.) the switching with respect to the nucleus they are bound to. In the "Before" panel the projectile is located at $(1,0,-5)$ for clarity.

standard 4b- and switching-CTMC approaches, considering a final time of integration of $2000 / v$ a.u., as previously stated. We have checked that the differences with those cross sections calculated with a final integration time of $500 / v$ a.u. are around $4 \%$, and therefore the calculation can be considered as converged. We have also analyzed the convergence with statistics estimating the standard deviation error of the probability as explained in [54], and we have found errors smaller than $3 \%$. These cross sections are compared to experimental data [26,28], previous 4b-CTMC [36,38], and semiclassical atomic close-coupling [31] calculations. Our 4b-CTMC cross section agrees with that of Dimitriou et al. [38], as it could be expected since these authors also employed the standard 4b-CTMC method. Both calculations are found to strongly underestimate the experimental cross section because of the inability of 4b-CTMC to describe a stable $\mathrm{H}^{-}$(see the illustration in Fig. 1). However, and surprisingly, the 4b-CTMC results of Olson [36] lie high above the experimental data, which is probably due to an excessively short integration final time in his calculations, as can be surmised from Fig. 1. On the other hand, our
switching-CTMC approach yields converging probabilities, and the associated cross section shows nice agreement with experiments [26,28]. The agreement with the close-coupling results of Wang et al. [31] is also remarkable.

This result encouraged us to address the study of the inverse reaction (2). In this case, we start the calculation in the $2 \times 3 \mathrm{~b}-\mathrm{CTMC}$ framework to describe the $\mathrm{H}^{-}\left(e_{1}, e_{2}\right)$ target. The two-electron initial distribution is the product of two microcanonical distributions, both located at the target center, involving the static model potential $V_{\text {mod }}$ to represent mutual electron-electron screening. In other words, each electron pair in the $2 \times 3 \mathrm{~b}-\mathrm{CTMC}$ calculation initially consists of two independent and equivalent electrons with energy -0.027 a.u. This can seem contradictory with the intuitive picture of $\mathrm{H}^{-}$, which usually involves the concept of inner and outer electrons. (Even if in quantum mechanics both electrons are equivalent.) Therefore, we show in Fig. 4 that there is no paradox: the mean monoelectronic energies, $\left\langle E_{i}^{(\mathrm{T})}\right\rangle$, computed by averaging $E_{i}^{(\mathrm{T})}=p_{i}^{2} / 2-1 / r_{i}$ over the $\mathcal{N}$ independent trajectories, approach -0.5 a.u., as expected, while the mean repulsion $<1 / r_{12}>$ is close to 0.5 a.u. The total electronic energy 


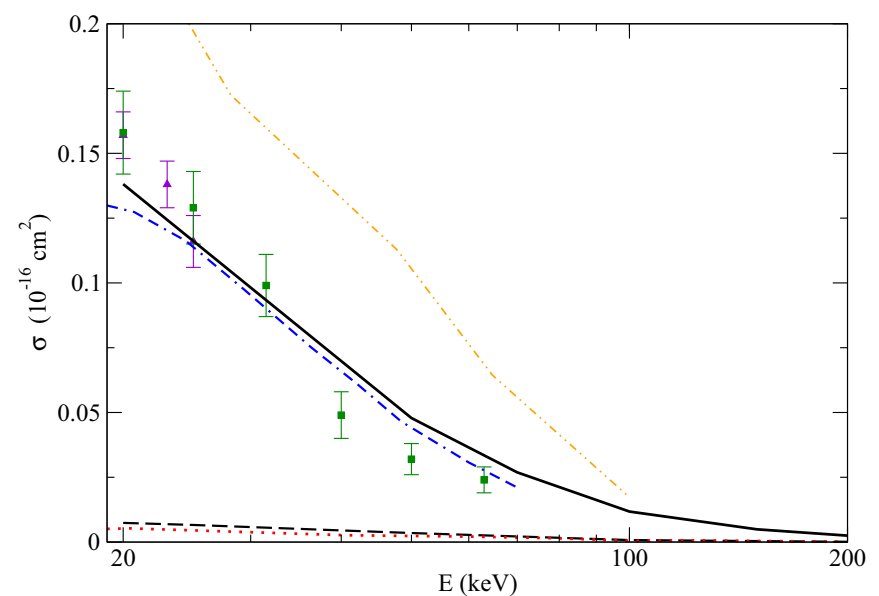

FIG. 3. Total cross section for negative-projectile ion formation [Eq. (1)] as a function of the incident energy $E$ in the laboratory frame. Present switching (-) and 4b (- -) CTMC calculations. Previous 4b-CTMC results from Dimitriou et al. [38] (. . .) and Olson [36] (- . - ). Two-center two-electron close-coupling calculations from Wang et al. [31] (- - - -). Measurements from Hill et al. [28] (ム) and McClure [26] (ם).

is thus $<E_{1}^{(\mathrm{T})}>+<E_{2}^{(\mathrm{T})}>+<1 / r_{12}>\approx-0.5$ a.u., close to the quantum-mechanical value $(-0.5027$ a.u.). Once the initial conditions are appropriately established, we numerically integrate the Hamilton equations of the two independent three-body systems, and we switch to 4b-CTMC calculation as soon as the active electron of one of the two threebody systems is released. This switch is opposite to the one illustrated in Fig. 2 and occurs in a similarly smooth way. Moreover, we show in Fig. 4 that the switch suitably describes on the electronic energy scale the process of direct

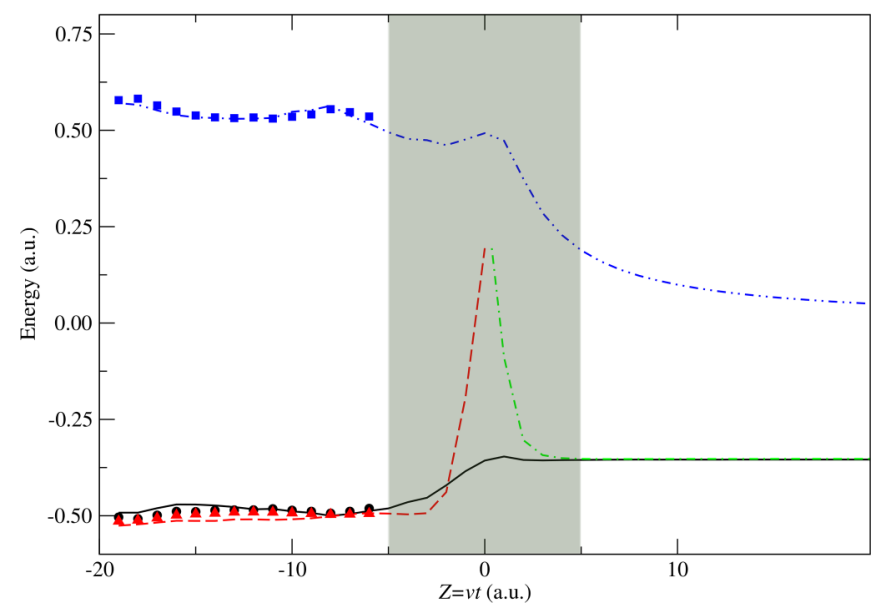

FIG. 4. Illustration of the temporal evolution of mean monoelectronic energies $<E_{1}^{(\mathrm{T})}>(-, \bullet),<E_{2}^{(\mathrm{T})}>(---, \mathbf{\Delta}),<E_{2}^{(\mathrm{P})}>(-\cdot$ $-\cdot)$ and mean interelectronic repulsion, $\left\langle 1 / r_{12}>,(-\cdots,-\mathbf{\square})\right.$ for a nuclear trajectory with $E=30 \mathrm{keV}$ and $b=1$ a.u. in $\mathrm{H}^{+}+\mathrm{H}^{-}$ collisions. The lines refer to averages over the electron pairs $\left(e_{1}, e_{2}\right)$ leading to direct neutralization, while the dots refer to averages over all $\mathcal{N}^{2}$ electron pairs. The shaded area indicates the region where the switching between $2 \times 3 \mathrm{~b}$ - and $4 \mathrm{~b}-\mathrm{CTMC}$ descriptions takes place.

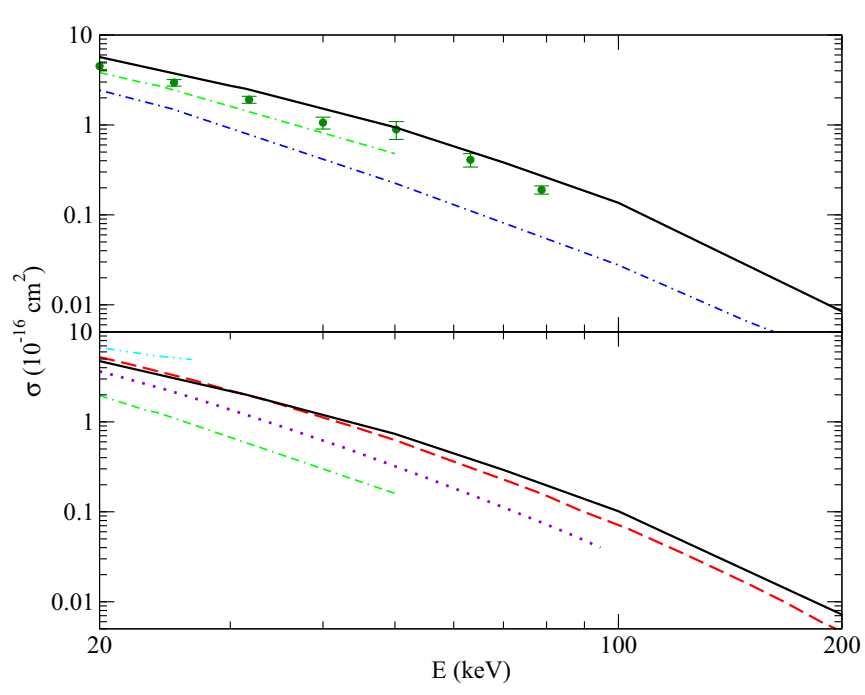

FIG. 5. Total cross section for mutual neutralization in $\mathrm{H}^{+}+\mathrm{H}^{-}$ collisions as a function of the collision energy $E . \mathrm{H}^{+}+\mathrm{H}^{-}\left(1 s^{2}\right) \rightarrow$ $\mathrm{H}(n l m)+\mathrm{H}\left(n^{\prime} l^{\prime} m^{\prime}\right)$ in the upper panel and $\mathrm{H}(n l m)+\mathrm{H}(1 s)$ in the lower panel. Present CTMC results obtained with the switching (-) and 3b-IPM-CTMC (- - -) approaches. Coupled-channel calculations from Shingal and Bransden [30] (- - - -), Wang et al. [31] (- - -), and Ling and Wang [55] (. . ). CB1-4B results from Mancev et al.[51] (- - -). Experimental results from Schön et al. [40] (•).

neutralization (2); we observe along the collision ( $E=30 \mathrm{keV}$, $b=1$ a.u.) a smooth transition from the initial condition, with $<E_{1}^{(\mathrm{T})}>\approx<E_{2}^{(\mathrm{T})}>\approx-0.5$ a.u., to the final situation where $<E_{1}^{(\mathrm{T})}>\approx<E_{2}^{(\mathrm{P})}>\approx-0.5$ a.u. and $<1 / r_{12}>\approx 0$.

The total cross section for mutual neutralization [Eq. (2)], obtained by means of the switching-CTMC approach, is plotted in the upper panel of Fig. 5. It is in good agreement with the experimental data of Schön et al. [40]. The improvement related to switching is gauged by the comparison with the cross section resulting from 3b-IPM-CTMC calculations. In such calculations, static screening is employed throughout the interaction and the neutralization probability is computed using the independent particle model $[14,15]$ as $2 p_{c} p_{e}$, where $p_{c}$ and $p_{e}$ are monoelectronic probabilities for capture and elastic+excitation processes, respectively. The neutralization cross section is drastically underestimated in the 3b-IPM framework. At low energies, the cross section issued from switching CTMC is close to the cross sections obtained by means of two-center atomic close-coupling calculations [30,31]. In the lower panel of Fig. 5 we consider the neutralization process restricted to production of target $\mathrm{H}$ into its ground state, i.e., $\mathrm{H}^{+}+\mathrm{H}^{-} \rightarrow \mathrm{H}(n l m)+\mathrm{H}(1 s)$. The cross section obtained by means of the switching approach is in very satisfactory agreement with the four-body boundary-corrected first Born (CB1-4B) results of Mancev et al. [51]. The two-center closecoupling calculations of Shingal and Bransden [30], which provide a satisfactory cross section for total neutralization, seem to overestimate the production of target $\mathrm{H}$ atoms in excited states. In our calculations, the mutual neutralization with target excitation processes account for $16.5 \%$ of mutual neutralization at $20 \mathrm{keV}$. It reaches a maximum value of $\sim 25 \%$ at around $120 \mathrm{keV}$ and decreases down to $15.5 \%$ at $200 \mathrm{keV}$. 
In conclusion, we have introduced a classical method which circumvents in a simple way the issue of artificial ionization in collisional processes involving two active electrons. This approach, referred to as switching CTMC, is based on the standard four-body procedure as long as the two electrons are not bound to the same nuclear center, and switching to three-body calculations involving a static screening description for both active electrons is applied as soon as they bind to the same ion. Switching from four- to three-body descriptions (and vice versa) may occur multiple times along the same nuclear trajectory. We have shown that the switching-CTMC treatment applies successfully to the benchmark collisions $\mathrm{H}+\mathrm{H}$ and $\mathrm{H}^{+}+\mathrm{H}^{-}$. For $\mathrm{H}+\mathrm{H}$, where the electrons are initially located on different centers, the approach provides an accurate description of $\mathrm{H}^{-}$formation, which cannot be described by applying the standard four-body
CTMC method. For $\mathrm{H}^{+}+\mathrm{H}^{-}$, with two active target electrons, the switching-CTMC method yields a much better description of neutralization processes than usual three-body treatments. In the near future, we plan to apply our switching-CTMC approach to ion-cluster interactions described in terms of underlying ion-atom collisions. We indeed recently showed that a simple description of electron correlation hinders an accurate treatment of some processes such as cluster anion formation [20]. The switching-CTMC approach should remedy this problem.

\section{ACKNOWLEDGMENTS}

This work has been partially supported by Ministerio de Economía y Competitividad (Spain) (Projects No. ENE201128200 and No. ENE2014-52432-R).
[1] R. Abrines and I. C. Percival, Proc. Phys. Soc. 88, 861 (1966).

[2] R. E. Olson and A. Salop, Phys. Rev. A 16, 531 (1977).

[3] C. Illescas and A. Riera, Phys. Rev. A 60, 4546 (1999).

[4] R. Dörner, H. Khemliche, M. H. Prior, C. L. Cocke, J. A. Gary, R. E. Olson, V. Mergel, J. Ullrich, and H. Schmidt-Böcking, Phys. Rev. Lett. 77, 4520 (1996).

[5] C. Illescas, B. Pons, and A. Riera, Phys. Rev. A 65, 030703(R) (2002).

[6] L. F. Errea, Clara Illescas, L. Méndez, B. Pons, A. Riera, and J. Suárez, Phys. Rev. A 70, 052713 (2004).

[7] P. J. Ho, R. Panfili, S. L. Haan, and J. H. Eberly, Phys. Rev. Lett. 94, 093002 (2005).

[8] S. L. Haan, J. S. Van Dyke, and Z. S. Smith, Phys. Rev. Lett. 101, 113001 (2008).

[9] P. Botheron and B. Pons, Phys. Rev. A 80, 023402 (2009).

[10] Y. Zhou, C. Huang, Q. Liao, and P. Lu, Phys. Rev. Lett. 109, 053004 (2012).

[11] C. Lemell, J. Burgdörfer, S. Gräfe, K. I. Dimitriou, D. G. Arbó, and X.-M. Tong, Phys. Rev. A 87, 013421 (2013).

[12] F. Cloux, B. Fabre, and B. Pons, Phys. Rev. A 91, 023415 (2015).

[13] F. Guzmán, L. F. Errea, and B. Pons, Phys. Rev. A 80, 042708 (2009).

[14] J. H. McGuire and L. Weaver, Phys. Rev. A 16, 41 (1977).

[15] H. Lüdde and R. Dreizler, J. Phys. B 18, 107 (1985).

[16] R. K. Janev and J. W. Gallagher, J. Phys. Chem. Ref. Data 13, 1199 (1984).

[17] M. B. Shah, C. McGrath, Clara Illescas, B. Pons, A. Riera, H. Luna, D. S. F. Crothers, S. F. C. ORourke, and H. B. Gilbody, Phys. Rev. A 67, 010704(R) (2003).

[18] C. O. Reinhold and C. A. Falcón, Phys. Rev. A 33, 3859 (1986).

[19] B. Bransden and M. McDowell, Charge Exchange and the Theory of Ion-Atom Collisions (Clarendon Press, Oxford, 1992).

[20] G. Labaigt, A. Jorge, C. Illescas, K. Béroff, A. Dubois, B. Pons, and M. Chabot, J. Phys. B 48, 075201 (2015).

[21] L. F. Errea, J. D. Gorfinkiel, C. Harel, H. Jouin, A. Macías, L. Méndez, B. Pons, and A. Riera, J. Phys. B 33, 3107 (2000).

[22] V. J. Montemayor and G. Schiwietz, Phys. Rev. A 40, 6223 (1989).

[23] C. L. Kirschbaum and L. Wilets, Phys. Rev. A 21, 834 (1980).
[24] A. E. Wetmore and R. E. Olson, Phys. Rev. A 38, 5563 (1988).

[25] J. S. Cohen, Phys. Rev. A 54, 573 (1996).

[26] G. W. McClure, Phys. Rev. 166, 22 (1968).

[27] A. B. Wittkower, G. Levy, and H. B. Gilbody, Proc. Phys. Soc. 91, 306 (1967).

[28] J. Hill, J. Geddes, and H. B. Gilbody, J. Phys. B 12, 3341 (1979).

[29] F. Borondo, F. Martín, and M. Yáñez, Phys. Rev. A 35, 60 (1987).

[30] R. Shingal and B. H. Bransden, J. Phys. B 23, 1203 (1990).

[31] J. Wang, J. P. Hansen, and A. Dubois, J. Phys. B 33, 241 (2000).

[32] D. R. Bates and G. W. Griffing, Proc. Phys. Soc. A 68, 90 (1955).

[33] R. A. Mapleton, Proc. Phys. Soc. 85, 841 (1965).

[34] K. Omidvar and H. Lee Kyle, Phys. Rev. A 2, 408 (1970).

[35] I. Mancev, Phys. Scr. 51, 762 (1995).

[36] R. E. Olson, J. Phys. B 12, L109 (1979).

[37] R. L. Becker and A. D. McKellar, J. Phys. B 12, L345 (1979).

[38] K. Dimitriou, K. Katsonis, and G. Maynard, J. Phys. IV (France) 10, 299 (2000).

[39] B. Peart, R. Grey, and K. T. Dolder, J. Phys. B 9, 3047 (1976).

[40] W. Schön, S. Krüdener, F. Melchert, K. Rinn, M. Wagner, and E. Salzborn, J. Phys. B 20, L759 (1987).

[41] W. Schön, S. Krüdener, F. Melchert, K. Rinn, M. Wagner, E. Salzborn, M. Karemera, S. Szücs, M. Terao, D. Fussen, R. Janev, X. Urbain, and F. Brouillard, Phys. Rev. Lett. 59, 1565 (1987).

[42] F. Melchert, S. Krüdener, K. Huber, and E. Salzborn, J. Phys. B 32, L139 (1999).

[43] H. Braüning, H. Helm, J. S. Briggs, and E. Salzborn, Phys. Rev. Lett. 99, 173202 (2007).

[44] S. M. Nkambule, N. Elander, A. Larson, J. Lecointre, and X. Urbain, Phys. Rev. A 93, 032701 (2016).

[45] F. Borondo, A. Macías, and A. Riera, Chem. Phys. 81, 303 (1983).

[46] D. Fussen and C. Kubach, J. Phys. B 19, L31 (1986).

[47] A. M. Ermolaev, J. Phys. B 21, 81 (1988).

[48] L. F. Errea, C. Harel, P. Jimeno, H. Jouin, L. Méndez, and A. Riera, J. Phys. B 26, 3573 (1993). 
[49] L. F. Errea, C. Harel, P. Jimeno, H. Jouin, L. Méndez, and A. Riera, Phys. Rev. A 54, 967 (1996).

[50] M. Stenrup, A. Larson, and N. Elander, Phys. Rev. A 79, 012713 (2009).

[51] I. Mancev, N. Milojević, and D. Belkić, Europhys. Lett. 103, 23001 (2013).
[52] H. M. Hedberg, S. Nkambule, and Å. Larson, J. Phys. B 47, 225206 (2014).

[53] J. D. Talman, Comput. Phys. Commun. 54, 85 (1989).

[54] F. Sattin and L. Salasnich, Phys. Scr. 58, 464 (1998).

[55] L. Liu and J. Wang, Chin. Phys. Lett. 24, 3115 (2007). 